\title{
66. A Case of Mosaic Ring Chromosome No. 22 with the Mental Retardation
}

\author{
By Suzue Kanata,*) Tetsuji Kadotani,**) Yoko Watanabe,**) \\ Noriko KUrosaki,**) Toshio Kumada,*) and Osamu ToI***) \\ (Communicated by Sajiro Makino, M. J. A., Sept. 14, 1987)
}

It has been well known that the chromosome abnormality on no. 22 leads a high susceptibility for the constitutional abnormality. Most of the abnormalities on chromosome no. 22 is the translocation. The translocation between chromosome no. 22 and no. 11 is one of the most frequent translocations in the population. The existence of ring chromosome no. 22, however, is rather rare.

During a chromosome survey of congenital defectives, a 30-year-old woman with the mental retardation was found to have a ring chromosome no. 22. Previously, the authors reported another case of a ring chromosome no. 22 (Kadotani et al. 1987).

Case report. The propositus was a 30 -year-old mentally retarded woman, $41 \mathrm{~kg}$ in weight, $150 \mathrm{~cm}$ in height. She was born to a 34-year-old mother and a 31-year-old father as the third child. There was no history of abortion, stillbirth, congenital malformations, mental retardation, exposure to the atomic bomb, and consanguinity in this couple. Her parents and her elder brother and sister were phenotypically normal.

At birth, she was in the state of asphyxia after 7 months of the eventless gestation. Her birth weight was $1900 \mathrm{~g}$. Her development was retarded at both physical and mental level. She walked alone at the age of 24 months, and she spoke a first word at the age of 36 months.

The remarkable clinical signs of this propositus were the mental retardation; idiot, speech disorders, seizure, slight ataxic gait, and kyphosis. The physical abnormalities were few.

Cytological findings. Chromosome slides were prepared in accordance with the standard blood culture procedure. Chromosome numbers were determined with 78 well-delineated metaphases. Karyotype analyses were made with the application of the conventional Giemsa staining and G-, C- and N-banding differential staining.

The chromosome constitution of the propositus derived from a conventional Giemsa specimen showed 46 chromosomes, and a ring-shaped G group chromosome was found in high frequency. On the analysis of G-banding method, it was revealed that the ring chromosome was one of no. 22 chromosomes. On the C-banding analysis, the presence of centromere was manifested. On the N-banding (silver staining: Bloom and Goodpasture 1976) analysis, the absence of the nuclear organizing region was revealed. This would suggest that a break occurred at p11 (Fig. 1). The G- and N-banding patterns suggested that the

*) Psychiatric Department, National Sanatorium Kamo Hospital. Kurose-cho, Kamo-gun, Hiroshima, Japan.

**) The Kadotani Medical Research Foundation. 1248, Saijohigashi, Saijo, Higashihiroshima, 724, Japan.

***) Chugokugeinan-Gakuen. Tadanoumi-cho, Takehara, Hiroshima, Japan. 

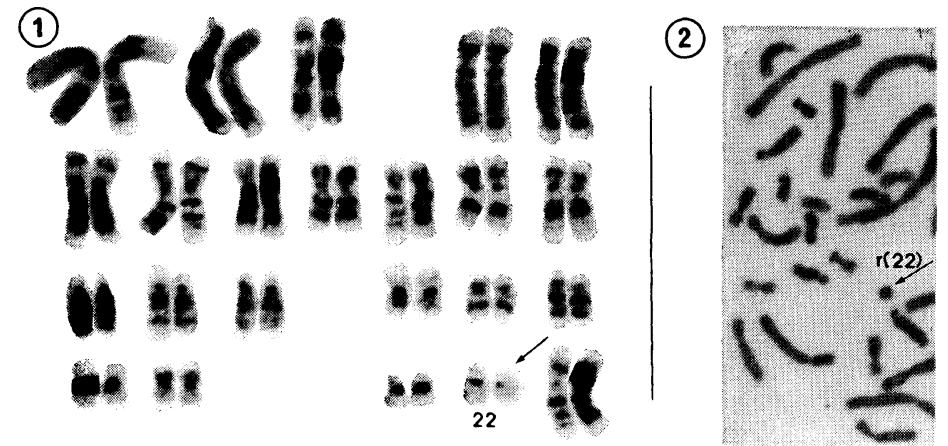

Fig. 1. (1) G-banding karyotype, and (2) partial N-banding metaphase of the propositus.

distal break of the long arm of no. 22 chromosome was at o13 (Fig. 1). Out of 78 cells counted, 9 cells $(11.5 \%)$ had $46, \mathrm{XX}$, and 69 cells $(88.5 \%)$ had $46, \mathrm{XX}, \mathrm{r}(22)$. Then the chromosome formula of the propositus was given as $46, \mathrm{XX} / 46, \mathrm{XX}, \mathrm{r}(22)$ (p11q13).

The chromosomal examinations of her parents and her siblings were not cooperated.

Remarks and conclusion. Although chromosome no. 22 is very small, the abnormality of chromosome no. 22 causes many diseases including malignancies such as chronic myelogenous leukemia, Burkitt's lymphoma, Ewing's sarcoma. Most of the abnormalities on the chromosome no. 22 result from the translocation. However, the ring chromosome no. 22 rarely happens. Previously, the authors reviewed 29 cases of the ring chromosome no. 22 (Brookfield and Walker 1976; Rethoré et al. 1976; Hunter et al. 1977; Howard-Peebles 1977; Palmer et al. 1977; Funderburk et al. 1979; Fryns and Berghe 1979; Fowler et al. 1980) and referred to the relation between clinical features and the ring chromosome no. 22 . The distinctive clinical features of the ring chromosome no. 22 were consisting of mental retardation with verbal delay, epicanthal folds, full eyebrows, large ears, arched palate and/or high palate, syndactyly, ataxic gait and seizure.

The case of us manifested some frequent features: the mental retardation, verbal delay, ataxic gait and skeletal abnormality, but the facial dysmorphism was poor. The poorly facial dysmorphism of the proband may be owed to the fact that she had a mosaic-typed ring chromosome no. 22 .

Acknowledgements. We are cordially obliged to Emeritus Professor Sajiro Makino, M. J. A., the senior director of the Kadotani Medical Research Foundation, for improvement of this manuscript. Financial aid from the Japan Academy is gratefully acknowledged here.

\section{References}

Bloom, S. E., and Goodpasture, C. (1976) : Hum. Genet., 34, 199-206.

Brookfield, D. S. K., and Walker, S. (1976) : J. Med. Genet., 13, 530-532.

Duncan, A. M. V. et al. (1987) : Cancer Genet. Cytogenet., 25, 169-174.

Fowler, G. et al. (1980) : Clin. Genet., 18, 274-279.

Fryns, J. P., and Van den Berghe, H. (1979): Hum. Genet., 47, 213-216.

Funderburk, S. J. et al. (1979) : Clin. Genet., 16, 305-310. 
Howard-Peebles, P. N. (1977) : J. Hered., 68, 268-269.

Hunter, A. G. W. et al. (1977) : Clin. Genet., 12, 239-249.

Kadotani, T. et al. (1987) : Proc. Japan Acad., 63B, 93-95.

Kaplan, J. C. et al. (1987) : J. Med. Genet., 24, 65-78.

Maeda, T. et al. (1977) : Hum. Genet., 35, 255-259.

Palmer, C. G. et al. (1977) : ibid., 14, 54-60.

Rethoré, M.-O. et al. (1976) : Ann. Génét., 19, 111-117. 